\title{
TELE'DRAMA-International sociometry in the virtual space
}

\section{Implementing Action Methods via Online Video Communication}

\author{
Daniela Simmons • Andrea Wilches
}

Accepted: 8 June 2021 / Published online: 10 August 2021

(C) Springer Fachmedien Wiesbaden GmbH, ein Teil von Springer Nature 2021

\begin{abstract}
This article aims to offer an overview of Tele'Drama as a method and its pioneering role in implementing action and experiential methods via online communication, as well as its purpose in applying J. L. Moreno's view on international sociometry. The main author of this article is the creator of the method, and believes that Tele 'Drama is a particularly important part of the future of action methods as it is also a bridge between locations and cultures.
\end{abstract}

Keywords Tele'Drama $\cdot$ Psychodrama $\cdot$ Sociometry $\cdot$ Action methods · Online

\section{Introduction}

Our move toward international sociometry is motivated by the state of affairs of our world, a situation about which behavioral scientists must help acquire knowledge if they are to discharge their universal responsibility to science, to humanity, to their nations, and to themselves. We are dedicated to the internationalism of science-not only in word, but also in action. The building of intercultural bridges presupposes the growth of a body of knowledge. (Moreno 1956, p. 1)

Tele'Drama is a method for offering action and expressive methods training, therapy, counseling and other interventions by using an online video conferencing approach. Created as a method and coined as a term by Daniela Simmons, Tele'Drama has been gradually developed and experimented with since Fall 2017. The term "Tele'Drama" (with an apostrophe between 'tele' and 'drama'), was cre-

Daniela Simmons, $\mathrm{PhD}(\bowtie)$

1014 E. Hwy 82, Ste 310, Gainesville, TX 76240, USA

E-Mail: danielatsv@gmail.com 
ated to provide an original meaning. Its etymology comes from the Greek têle $(\mathrm{T} \eta \lambda \varepsilon)$, "at a distance" and drama $(\delta \rho \tilde{\alpha} \mu \alpha)$ "action." The apostrophe between 'tele' and 'drama' indicates the presence of various action methods offered at the institute: Psychodrama, Sociometry, Sociodrama, Bibliodrama, Playback Theatre, Theatre of the Oppressed, Dynamic Theatre, Dramatic Multiplication, Expressive Therapies (including Dance and Movement, Art, Drama, Poetry, Music Therapy, Organizational Consulting, Supervision, etc.) The term Tele'Drama also refers to Moreno's term of 'tele', "an inter-personal experience, growing out from person to person, ... and gradually developing the sense for inter-personal relations" (Moreno and Jennings 1938, p. 342).

Due to the Covid-19 pandemic, online formats have been the primary or only possible format available for meetings, therapy sessions and trainings since February 2020. However, Tele'Drama was created a long time before this current pandemic. The method faced strong resistance at first; action methods have been traditionally perceived as face-to-face applications. And yet, at the same time, physical meetings have become challenging to many due to the increasing cost of travel, travel restrictions to certain locations, physical or mobility impairments, busy schedules and time restraints, family obligations, and other reasons. Pre-pandemic, professionals in the action methods fields mostly attended only local conferences and events. The Tele'Drama method was offered as an opportunity to include people from various locations around the world, overcoming restrictions and distances, enabling people from many countries to learn from each other and to exchange the richness of their cultures.

Since its inception in 2017, Tele'Drama developed from a project into the International Tele'Drama Institute (ITI) with a strong team of organizers and trainers. From the beginning, it has utilized Zoom video-conferencing software with visual and sound technology for creating virtual rooms, adapting and modify classical action methods' techniques to be relevant to this online format wherein directors and their groups (or individual clients) are able to communicate, self-express and experience as if they were together in the same physical location even while joining in from locations around the world. The pressures of the global pandemic have, in fact, stimulated Tele'Drama's continued evolution, so that it now can provide online educational resources in various action methods, as well as professional mentoring, business leadership development, and team building.

\section{Historical roots}

Moreno was the first to use motion pictures and television to apply psychodrama in the treatment of married couples, families and groups (Moreno and Fischel 1942, p. 7). Working at Saint Elizabeth's Hospital in Washington DC, Moreno asked that the psychodrama group work should be done on television (Moreno 1968, p. 106). In 1964 at the Camarillo California State Hospital, Moreno was televised weekly on closed circuit TV while conducting psychodrama sessions (Moreno 2006a). In 1966 he conducted a seminar at the State Hospital in Pueblo, Colorado, and asked his psychodrama session to be televised on closed circuit TV as a teaching module for the 
hospital staff (Moreno 1968, p. 109). Another psychodrama session utilizing closed circuit television took place at the Southern Florida State Hospital in 1968. Zerka Moreno wrote, "Psychodrama on the television screen becomes an excellent introduction to the psychodramatic method" (1968, p. 175). Hare and Hare (1996), noted that, "Moreno recommends the use of television, motion pictures, and simultaneous psychodramatic re-trials in many parts of the world to make a mass co-experience possible" (p. 63). Moreno (1963), defined Treatment at a Distance, Television Psychodrama, Therapeutic Motion Pictures and Telephone Therapy; he wrote, "If the technologist can replace the 'audio telephone' with a 'video telephone,' the patient and therapist could not only hear but also see each other in action" (p. 117).

Marcia Karp, an early student of Moreno and a prominent international psychodrama trainer, remembers Moreno saying in the 1960's that, "One day we could share situations all over the world by simply pressing a button. A fisherman in Norway, for example, could share his family dilemma about his son, with a father in Japan who has a similar issue" (2020, personal communication).

\section{Research and evaluation at the International Tele'Drama Institute (ITI)}

Research sociometry During his college years and early psychiatric practice in Vienna, Moreno investigated interpersonal choices and attractions, and various relations in which people were involved. Moreno's work led to the creation of Research Sociometry, exploring group dynamics and social relationships. The Tele'Drama institute includes sociometric measurement at the beginning, during and at the end of our sessions, using spectrograms, locograms, sociometric test with specific criteria and sociograms, especially adapted for virtual environments.

Encounter \& tele Moreno's research on relations was also based on the "Encounter." Early in his college years in Vienna, Moreno and a colleague started a group for immigrants and refugees called "The Religion of the Encounter." Several years later Moreno wrote about "encounter," describing it as a meeting between the self and the inner self, or the other, or God. Later he added the important element "tele," defining it as the engine of human relations and the encounter. ITI's work is based on Moreno's idea of encounter between a director and the group; between the participants who are present, as well as with significant others who are not present (through use of auxiliary actors and the "empty chair" technique); and encounters with self in the past, present or future.

Spontaneity and creativity Moreno defined the whole universe as intrinsically creative. He believed that low creativity and low spontaneity are a result of high anxiety. The Tele'Drama Institute has based its program on exploring and stimulating spontaneity and creativity as the path to new cultural conserves. 


\section{Assuring safety in hosting online psychodrama sessions}

While some online webinars are designed to allow attendees to view only the presenters, ITI's meetings in "Zoom rooms" are programmed for events to be collaborative, with all participants able to see everyone in attendance. The Tele'Drama experiential modules, wherein group members can warm up, apply action techniques and share their experience openly. Zoom rooms also provide participants the ability to work in small breakout groups, complete group projects, and create an environment as close as possible to one in a physical space. Safety is most important in directing action methods sessions. The ITI professionals offering these sessions need to know how to assure safety, from appropriate Zoom meeting settings and technology to ethical and procedural recommendations for handling sessions, as well as safely and professionally directing and responding to the group's dynamics and individual member's catharses.

In present times, when it has been necessary to direct psychodrama sessions online, assuring safety can be especially challenging simply because the very concept of virtual group work is new; the answer to anyone's question of how to assure safety may be unknown due to insufficient knowledge, experience, or ability to access technology. In addition, the director often needs to multi-task and handle unusual issues that will undoubtedly occur in any session.

With the increasing popularity of the Zoom Meeting services, a new occurrence called 'Zoom bombing' appeared. The term refers to unwanted and disruptive intrusion into a teleconferencing session by an unaffiliated person or persons, something that can be exceptionally harmful and traumatic to the members of a group experiencing a therapeutic process. Therefore it is imperative that Zoom meeting hosts need to be skilled in programming and managing the pre-meeting and in-meeting settings such as: requiring passcodes to enter events, using "waiting rooms" prior to allowing participants to join events, and when necessary, the ability to mute an attendee's audio and video screen, disallow file sharing or the ability to add written comments in the "chat" area, and the like.

The Tele'Drama modules are influenced by the group dynamics. Working with online groups is a process similar to working with groups in the physical space. During online sessions group members have an opportunity to understand more about themselves and others, to explore past, present or future events in a safe environment, and to develop new perspectives.

Participants are invited to share about themselves as much as they feel comfortable, however, the more open they are the better the experience they will have. They might be sharing information in their group that is sensitive and personal, and it is important that they feel comfortable in the online environment. Group members are asked to agree to a confidentiality requirement in online group sessions.

The groups at the Tele'Drama institute are international, bringing the richness of various cultures and experiences to all group events. Respect for cultural and individual (and role) differences are critical, including those differences based on age, gender and gender identity, sexual orientation, race, ethnicity, culture, national origin, religion, disability, language and communication, and socioeconomic status. The sessions are unique to those who attend and are not recorded. All participants 
are asked to refrain from taking pictures of the group and sharing it with others or on social media, without the consent of the whole group.

Participants are asked to attend while using a quiet and safe place with no other people present in their near-by physical space, if possible. Using headsets is the safest way to participate if there are other people in the same room, however headsets can interfere with free and easy movement. Participants are asked to situation themselves so that they have a wall behind them rather than open space where other people or animals might appear; this is easy to arrange prior to groups beginning, and contributes to safety as the other group members are not distracted by extraneous movement or sound. Having sufficient indirect lighting so participants can be easily seen also contributes to the director's and group members' ability to see each other and follow the expressed emotions. Video cameras need to be 'on' during the entire session for better cohesion, closeness and connection between the members, and for director's ability to observe and handle possible abreactions among the group members. Participants are invited to refrain from going on 'mute' (disengaging their audio or video feeds), for the best experience of spontaneity and creativity. "Gallery view" allows participants to see the whole group at once, depending on the number of participants and the size of participants individual screens.

Warming up participants to the online environment starts well before the session begins. All participants are invited to log in $10 \mathrm{~min}$ before a session is scheduled to start and to stay throughout the entire session, including the closure. The director invites them to a new perspective that, even though everyone is physically in separate locations, the group is together in the same virtual space; that entering the Zoom meeting is like entering a physical room where people are sitting in a circle, having a chance to warm up to each other, to work on an issue, to share, to be playful and expressive, spontaneous and creative; that physical distance is not an obstacle and technology can support interpersonal relationships.

It is important that the group members be informed about certain risks. For example, during the session, they may remember unpleasant events or feelings. If these distressful emotions arise, they should be encouraged to talk both with the group and the group facilitator and to refrain from just leaving the meeting and dealing on their own with their experiences.

\section{Tele'Drama as methodology and international sociometry}

Moreno believed that "a truly therapeutic procedure must have no less objective than the whole of mankind" (Moreno, 1953, p. 1). His main philosophical concept is on the aim of implementing Sociatry-the healing of the societies (Moreno 2006b). As an innovative expansion of Moreno's methodologies of international psychodrama, sociometry, and sociatry, Tele'Drama supports his goal of the healing of societies. The uniqueness of Tele'Drama as a method lies in its ability to create and engage both global community and generate a transformative global culture. Tele'Drama nurtures a global community based on characteristics such as mutual respect, reciprocity, trustworthiness, and empathy. Tele'Drama as a methodology builds respect by giving global voices an equal hearing, advances empathy based 
on the feedback and dialogue between presenters and participants. Empathy is not only a feeling of caring and support for another, but a validated sense of being cared for and supported. Tele'Drama encourages and builds empathy with the multiple cultural backgrounds and worldviews that are likely to be present in online formats. This empathy, in turn, builds a culture based on commonalities of the broader global culture, of all humans rather than those priorities of this or that in-group. In this way, Tele'Drama has a major role to play in building our collective "immune system" that protects our personal and community health and well-being.

\section{Implementing action methods in the online settings}

Moreno was often visiting people's homes, directing psychodrama with an entire family and any visiting friends (Zuretti, 2020, personal communication). In the virtual world of Zoom online meeting rooms, facilitators lead groups in an imaginative process of visiting each other's homes, seeing the participants' video images on the screen as windows into a different part of the world and individuals' homes. Leaders also encourage participants to imagine stepping into the screen, entering the virtual space where they will be experiencing together, exploring the group process and leaving everything in their actual space behind for the time being.

Moreno believed that the psychological truth is often more real to people than the factual events that occur, because that is their truth, from their perspective. He defined this interpretation as poetical or psycho-dramatic truth, because this truth is the only one existing, according to Moreno, in human consciousness. Moreno (1965), wrote that psychodrama includes experiences "beyond reality," or what he termed "surplus reality." The surplus reality is a "gain" for the protagonist who is able to bring to the psychodrama stage imaginary scenes, images and people (p. 211). This philosophical concept needs to be implemented in the online group work, where the virtual space becomes a "psycho-dramatic truth," and the group process online is the true reality.

According to Zerka Moreno, in the reality of our lives there are "invisible dimensions" that we have not been able to completely express or experience (Moreno et al 2000, pp. 212-213). In Zoom meeting rooms, directors help group members explore the invisible dimensions of their virtual space and their images as holding space therein. For example, participants might be asked to imagine touching: holding hands, providing physical support with outstretched arms, and reaching out toward others through their computer cameras are ways to engage cultural and behavioral perceptions within a virtual experience. In contrast to the accustomed physical demonstrations of empathy, support or gratitude, in a virtual online space touch is expressed through a psychological perception, and can induce a comparable meaning and expression of physical communication.

Moreno (1965), believed that psychodrama and other action methods "brings the entire cosmos into play" (p. 211). This concept applies to the virtual reality of the online sessions, where the participants can be physically located thousands of miles apart, but psychologically are together in the same meeting space, involved simultaneously "into (the) play." Implementing psychodrama and other action methods 
online is beyond time, including people from various time zones; and does not depend on a certain physical location, but on the creation of group cohesion in the virtual space.

A strong warm-up phase and exploration of the groups' sociometry helps participants in an online session to relax, connect and trust each other, as well as trust the director. Helping participants to be fully present and in the here-and-now of the Zoom meeting is one of the most important tasks of the director in order to hold a successful session. The warm-up process starts even before the session begins, usually in an email warmly welcoming participants and providing an overview of guidelines about the upcoming process. This warms individuals up to the perception that the online environment will be close to one in a physical place, and helps them to set positive expectations. When the participants arrive in the "Zoom room," they need to receive another warm greeting, and experience activities and communication that will engage their attention immediately.

The need for confidentiality is addressed at the beginning of each session. Participants are expected to commit to being in a quiet and safe physical space without other people being seen or heard by the group members. A critical part of each online session is exploring the sociometry and the tele, or interpersonal connections, within the group, helping group members to connect and warm up to the "hereand-now." For example, spectrograms are applied through physical hand and arm demonstrations, using the dimensions of the small screen to help determine intensity of feelings or frequency of behaviors; locograms are applied through shared or projected virtual background images or props; sociometric tests and sociograms are applied using paper and markers, then sharing with the whole group by implementing additional apps such as screen-sharing and white board. A "step-in" sociometric exercise can be implemented by asking the group members to use their physical space, or to transform it to "Raise your hand if ..." exercise. The "Who like me ..." exercise, inviting participants to ask questions that are valid to themselves, helps the director to open up dialogue for personal sharing and connecting on a deeper level. Exercises for reflective listening and constructive communication enhance participants' attention to the process and the other group members.

To help group members feel safe when emotions are aroused, participants are invited to have personal objects nearby that create a "comfort place," with an awareness of the objects' meaning and availability as a support when needed. Group members are encouraged to take care of their physical needs and impulses, moving when and how as needed: invitations to sit or stand comfortably, reminders to breathe and relax - all of these reminders help participants to stay present and engaged. Strategies for creating interesting and enjoyable environments from the beginning of a session include imaginary activities for sensory stimulation (movement, touch, sound, sight, etc.), implementing shared images to invite meaning-making, and use of ambient sound and music as auditory warm-ups; all will nurture group participation and cohesion.

Implementing Zoom's application for breakout rooms allows group members to work and share in dyads or in small groups, and is a useful tool in creating interpersonal processes similar to those in the physical space. This requires the director or an assigned host to be well trained in using the application to avoid 
confusion and, thus, a drop in confidence of some group members. The director could choose to visit the small groups or to wait in the main Zoom room until all groups are brought back into the main room. Broadcasting reminders to the breakout rooms about the remaining time, and allowing a minute or two for participants to return after the breakout rooms have been closed greatly helps the participants to transition smoothly from the small group back to the large one.

There are three general approaches for choosing a protagonist: sociometric choice by the group, self-choice, and choice by the director. In the online environment, the three approaches remain the same. Sociometric choice by the group and self-choice can be done in variously creative ways, using self-measuring readiness through, for example, a show of hands, or body position or distance in relation to the computer's camera; as well as using paper and pencil or raising a hand to indicate whom participants are choosing for protagonist, and allowing group members to have an encounter (e.g., a brief empty chair vignette, or interview).

Scene setting contributes greatly to the experience of the here-and-now, allowing the protagonist to bring the outside world into the virtual meeting. The director asks the protagonist to identify the scene's location and to actively demonstrate it in their personal space (i.e., describing and "showing" how the scene looks and feels). They are asked to identify the sounds, odors, light, colors in the space; describe what is on the floor, on the walls, on the ceiling; detail the furniture, the decorations, and the like. For best effect, the whole group can be involved in the scene setting, by participants holding a role, creating an image, a sound, assisting in concretizing the time of and participants in the protagonist's scene. The director can ask the protagonist to walk in their space, to express their emotions, to examine the scene such as the texture on the floor or on the walls; to imagine "visiting" different parts of the scene, for example; opening drawers, a book or an album, changing the channels on the TV, or opening or closing the curtains.

Applying various psychodrama techniques in online psychodrama and sociodrama involves a director's spontaneity and creativity in choosing among variations, depending on the group, the protagonist and the director's comfort level. For example, the Empty Chair technique can be applied using an image of a chair and inviting the group's imaginary implementation of it; or asking participants to stand behind their actual chair, pull up another chair, or open a space next to them if they are sitting on a couch.

The Role Reversal technique is best applied in its original forms. The original form, called 'in situ' (Moreno 1959, p. 5); or "proper" (Moreno 1955, p. 88), is about two people, both present at the session and reversing roles with each other. The second form is called the "incomplete" or "classical" role reversal (Sabelli 1984, p. 139), when one of the people in the encounter is absent and is represented by auxiliary actor or an empty chair. Other forms of role reversal can be applied as well, if the group is small and the director is experienced in online interventions.

While the Zoom meeting application will allow participants to "mute" their video feed that would indicate being "off stage," the Tele'Drama method does not support having only the protagonist and the auxiliaries with video on, asking the audience to turn off their cameras. Even though that can be an easier process, the director will be unaware of possible abreactions among the group members if they are unseen. 
Instead, the Tele'Drama method easily identifies what members are in specific roles (vs. being an audience member) by the director asking group members to indicate their role in writing as a name tag, tor use various colors of scarves or props to identify the role they are playing, as well as particular expressions through posture, facial and oral identifies. In addition, auxiliaries are reminded to name their roles as they engage with the protagonist, for example, "As Mother I want to say ..."; "I am the Anger and I feel ..."

Doubling techniques can be implemented in many easy ways, from group members simply raising a hand or two fingers to identify readiness to double a protagonist or stating, "I want to double," or using the technical "meeting reactions" with the person who wants to double extending a hand as if they are placing their hand on the protagonist's shoulder and the protagonist reciprocating by placing their hand on their own shoulder to accept the connection. For implementing a "two voice doubling" (Morgan and Rinvolucri 2008, p. 1), the protagonist's image situated in the middle of the screen (programmed by the director or Zoom host), with the two auxiliaries on either side, talking softly. The protagonist can measure with their hands the intensity of feelings in each voice. In international groups where participants speak different languages, a "linguistic double" can be invited into the scene to help supply words or clarify meanings with which the protagonist is having difficulty. (Note: Sound in Zoom rooms can be distorted or interrupted unless the system is programmed for specific ambient environments; adjustments can and should be preprogrammed before the session begins, if possible.)

The Mirroring technique provides protagonists the sense of "stepping outside" of their psychodramatic scene, so that the protagonist can see the action in a mirror position to expand their perspective about a given situation. In the virtual room, the director invites the protagonist to choose a group member to stand in their place inside the scene, and to pull their chair back or to shift their body away from the screen to increase a sense of distance from their initial position.

\section{Tele'Drama and diversity}

As has been mentioned before, Moreno's concept of International Sociometry not only has to do with the construction of a social matrix that intertwines humanity as a whole for the building of a better world, but also with the construction that each one of us can chose to make our own matrix within us, so that this process becomes interactive. Tele'Drama as a method uses technology as a catalyst for this process, and offers a safe environment for equality and free expression; thus, fundamental for this to be possible, people need to feel they are within a space of containment and acceptance.

The universal premise is that we are all born free and equal, and everyone has the right to have their voices heard; this is why Tele'Drama promotes respect for each others' opinions throughout all group activities, and encourages participants to express themselves in whatever way they are able and feel comfortable, without imposing or pressuring anybody to do, express or say anything they do not feel like saying or doing. 
As stated in the Article Two of the Universal Declaration of Human Rights:

Everyone is entitled to all the right and freedoms set forth in the Declaration, without distinction of any kind, such as race, color, sex, language, religion, political or other opinion, national or social origin, property, birth or other status ... (United Nations 1984)

Diversity is not something that divides us; on the contrary, it is something that enriches the group process; it allows us to grow and expand our beliefs so that we can start building the fundamentals of the matrix of social identity and getting to know our differences while also finding in them the strengths of a common thread, moving from individuality towards collectivism. In his book Words of the Father (1941), Moreno works on the idea of a transpersonal view of mankind, that includes, of course, acknowledging diversity and stimulating everyone to become an active creator of their own reality and thereby the co-author of social change. "We are all bound together by responsibility for all things, there is no limited, partial responsibility. And responsibility makes us automatically creators of the world" (as cited in Nolte 2014, p. 179). Tele'Drama takes an active role in this responsibility, and is committed in promoting the construction of a world in which we can all have an active and equal presence.

\section{Conclusion}

With this article, the authors introduce the history, current development and specifics of the Tele'Drama method and the projects of the International Tele'Drama Institute (ITI). The method was created in Fall 2017, and was developed through experimentation with the delivery of various modalities and techniques. The main purpose of the method is to expand international sociometry, training in practical applications of action and expressive methods, group counseling and other interventions through online video conferencing applications.

\section{References}

\section{Cited literature}

Hare, A.P., \& Hare, J. R. (1996). J.L. Moreno. Thousand Oaks: SAGE.

Moreno, J. L. (1941). Words of the Father. Beacon: Beacon Press

Moreno, J.L. (1956). Forward. International Journal of Sociometry. Beacon: Beacon Press.

Moreno, J. L. (1963). The actual trends in group psychotherapy. Group Psychotherapy, 16, 117-131.

Moreno, J.L. (1965). Therapeutic vehicles and the concept of surplus reality. Group Psychotherapy, 18(4), 211-216.

Moreno, J.L. (2006a). Television videotape and psychodrama. American Journal of Psychiatry, 125(10), $1453-1454$.

Moreno, Z. (1959). A survey of psychodramatic techniques. Group Psychotherapy, 12, 5-14.

Moreno, Z. (1968). Psychodrama on closed and open circuit television. Group Psychotherapy, 11(2/3), $106-109$.

Moreno, Z. (2006b). The quintessential Zerka. New York: Routledge.

Moreno, J., \& Fischel, J. (1942). Spontaneity procedures in television broadcasting with special emphasis on interpersonal relation systems. Sociometry, 5(1), 7-28. https://doi.org/10.2307/2784951. 
Moreno, J.L., \& Jennings, H. H. (1938). Statistics of social configurations. Sociometry, 1(3/4), 342-374.

Moreno, Z., Blomkvist, L., \& Rutzel, T. (2000). Psychodrama, surplus reality and the art of healing. New York: Routledge.

Morgan, J., \& Rinvolucri, M. (2008). Doubling - a psychodrama technique that has been successfully transposed to language teaching. Humanizing Language Teaching, 10(2), 1.

Nolte, J. (2014). The philosophy, theory and methods of J.L Moreno: the man who tried to become God. New York: Routledge.

United Nations (1984). Universal declaration of human rights. https://www.refworld.org/docid/3ae6b3712c. html. Accessed 11 March 2021.

\section{Further Reading}

Moreno, J.L. (1969). Television videotape and psychodrama. American Journal of Psychiatry, 125(10), 1453-1454.

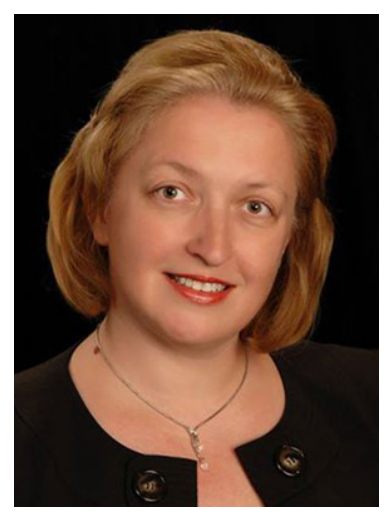

Daniela Simmons is a Certified Trainer, Educator, Practitioner (TEP), Supervisor in Psychodrama, Sociometry and Group Psychotherapy through the American Board of Examiners (ABE). She is the creator of the Tele'Drama Method and founder of the International Tele'Drama Institute (ITI). President, American Society of Group Psychotherapy and Psychodrama (ASGPP)

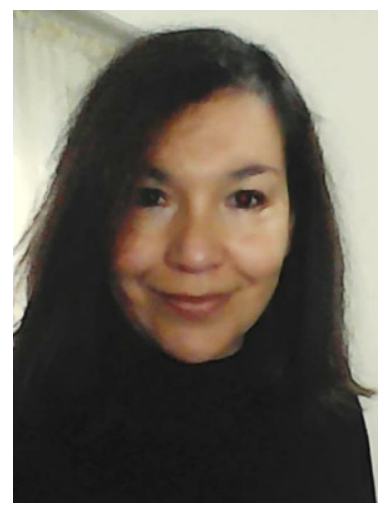

Andrea Wilches was born in Colombia where she graduated as a psychologist, in 2004. Since 2008 she lives, studies, and works in Buenos Aires, Argentina. In 2010 she completed her Psychodrama training and was given the opportunity to work as a trainer in the Psychodrama Pavlovsky Institute. She has been a trainer at the International Tele'Drama Institute (ITI) since the Spring of 2020. 